\title{
Sprachtechnologie für die Strukturierung digitaler Information
}

\begin{abstract}
Der Beitrag erläutert die Grundideen und Potenziale einer konsequenten hypermedialen Informationsvernetzung im WWW. Dazu werden Verfabren der automatischen Hyperverknüpfung vorgestellt, die Rolle der Sprachtechnologie in diesem Zusammenhang diskutiert und die Bedeutung der XML-Standards für die Verwirklichung einer dichten Hypervernetzung erklärt.
\end{abstract}

\section{Einleitung}

Das Internet vernetzt die Computer der Welt. Das World Wide Web vernetzt die weltweit zugängliche Information auf diesen Computern. Die Grundlage des WWW ist das Konzept des Hypertextes. Über Hyperreferenzen, die vom Browser als Sprungbefehle interpretiert werden, navigieren sich die Benutzer durch den unüberschaubaren Datendschungel.

Das Konzept des Hypertextes ist heute im Web aber nur partiell realisiert und selbst die vorhandenen Möglichkeiten zur Informationsvernetzung werden nur rudimentär genutzt. Daher ist die Information auf dem Web auch nur äußerst grob strukturiert. Gegenwärtig nimmt die Information auf dem WWW schneller zu als die mühsam von Hand geschaffenen Strukturierungen.

Eine zweite Welle der Hypertextrevolution baut sich auf. Durch drei bisher noch parallel laufende Entwicklungen entsteht die Basis für eine konsequentere Nutzung der hypermedialen Verknüpfung.

1. Im Informationsmanagement und zunehmend auch im Wissensmanagement werden Bedürfnisse erkannt und formuliert, die sich nur durch eine dichtere Vernetzung von Information erfüllen lassen.

2. Die Sprachtechnologie schafft die Möglichkeiten für eine weitestgehende Automatisierung dieser dichten Vernetzung, die auch viel zu aufwendig ist, als dass sie allein durch menschlichen Einsatz bewältigt werden könnte. 
3. Die gegenwärtig entwickelten neuen Standards für das WWW (XML im Verbund mit XLink, XPointer, XSL und RDF) werden die hypermediale Strukturierung der Information viel besser unterstützen als die bestehende HTML-Plattform.

In meinem Beitrag will ich die Grundideen und Potenziale der neuen Technologien an Beispielen erläutern. Zum Einstieg werde ich das Konzept der dichten hypermedialen Vernetzung skizzieren. Dann werde ich Funktionalitäten und Anwendungen schildern, die durch die automatische Hyperverknüpfung möglich werden, und dabei auch einige neue Anwendungsideen vorstellen. Anschließend wird die Rolle der Sprachtechnologien bei der Realisierung der Anwendungen diskutiert. Hervorgehoben wird in diesem Zusammenhang die Technologie der Informationsextraktion. Außerdem soll die Bedeutung der künftigen XML Standards für die Verwirklichung einer dichten Hypervernetzung erklärt werden. In dem abschließenden Ausblick werden Konsequenzen für die weitere wissenschaftlich-technologische Entwicklung vorhergesagt.

\section{Hypertext, Internet und dichte hypermediale Vernetzung}

Die Idee des Hypertextes wird Vannevar Bush (1945) zugeschrieben. Der frühere MIT Präsident und Director des Office of Scientific Research and Development entwarf in einem Artikel im Atlantic Monthly die Vision eines Systems, das das menschliche Gedächtnis ergänzen sollte. In dem System Memex sollte der Benutzer große Mengen von Büchern, Papieren und eigenen Aufzeichnungen speichern können, die durch Referenzen so vernetzt sein sollten, dass man ohne Suche assoziativ von Dokument zu Dokument springen kann. Da es 1945 keine Computer gab, die größere Mengen Daten verwalten konnten, schlug Bush als Medium für die Realisierung seines Wissensspeichers Microfilm-Folien mit einer speziellen Lesemaschine vor.

Der Begriff Hypertext stammt von Ted Nelson (1965), der seit circa 1963 an seinem eigenen System Xanadu zur Herstellung, Verwaltung und Lektüre von nichtlinear verknüpften Texten arbeitet. Nahezu gleichzeitig mit Nelson begann auch Doug Engelbart, der Erfinder der Maus, am Stanford Research Institute (SRI) mit der Konstruktion eines Systems namens Augment, das Hyperverknüpfung, Maus- und Fenstertechnologie verbindet, um den Menschen bei der kreativen Arbeit zu unterstützen. (Engelbart 1963). Ihren Siegeszug trat die Idee der Hypermedien an, als sie von den Schöpfern des WWW, T. Berners-Lee und R. Cailliau, technisch umgesetzt wurde, um Informationen, die verteilt auf dem Internet liegen, wirksam zu vernetzen. Die Umsetzung beruht auf einer Kombination der Annotationssprache (HTML) mit einem Netzwerkprotokoll (HTTP), das 
den Abruf der Dokumente aus einem Browser heraus ermöglicht. Die Hyperverknüpfungen sind funktionale, in den Text integrierte (inline) Referenzen auf ganze Dokumente. Zwar kann man innerhalb eines Zieldokuments auch an vorher markierte Zielorte springen, geladen wird auf jeden Fall das ganze Dokument.

Nun möchte ich behaupten, dass nicht nur die Hypertext-Idee erst durch ihre Verwendung auf dem Internet zum Durchbruch gelangte, sondern dass auch das Internet das Konzept der hypermedialen Verknüpfung brauchte, um zum Massenmedium zu werden. Die Technologie zur Vernetzung voneinander weit entfernter Rechner gibt es seit 1957 im ARPANET und später auch in anderen Vorläufern des Internet. Seit den achtziger Jahren ist das Internet mit den Protokollen TCP/IP das weltweite Netz. Als 1989 das WWW entwickelt wurde, umfaßte das Internet bereits 150.000 Rechner.

Warum hat es dann solange gedauert, bis das Internet von einem Werkund Spielzeug der technologischen Avantgarde zu einem Alltagsinstrument wurde? Sicher haben die gesunkenen Hardwarepreise und die daraus folgende größere Verbreitung des PC zur Explosion des Internet beigetragen, der wichtigste Grund für den Erfolg des Netzes der Netze ist jedoch die Informationsvernetzung. Ist das Internet ein Netz von Maschinen, die Informationen speichern und verarbeiten können, so ist das WWW die Vernetzung der zugänglichen Information auf diesen Maschinen. Die Knoten des Internet sind Computer, die Knoten des WWW sind Informationseinheiten, die auf diesen Computern gespeichert sind: Texte, Begrif$\mathrm{fe}$, Bilder, Filme und Klänge. Erst durch die Vernetzung der Information und durch die Möglichkeit, komfortabel per Mausklick durch das Netz zu navigieren, konnte das Internet zum Massenmedium werden.

Zwar gab es auch schon vorher Zugänge zu zentralen Datenbeständen via Datenfernübertragung - Kontostände, Flugverbindungen, Zimmerreservierungen und Fahndungslisten konnten über besondere Schnittstellen von speziell eingearbeiteten Fachkräften abgefragt werden. Die Technologie des WWW hat dafür gesorgt, dass heute jeder Benutzer eines Internetfähigen Computers solche Daten und viele andere Informationen bequem über den leicht zu bedienenden WWW-Browser auf den Bildschirm holen kann. Nur wegen ihrer einfachen intuitiven Bedienbarkeit wächst das WWW, die größte Datenbank der Welt, heute sehr viel schneller als jede andere Informationssammlung. Klickend navigieren Kinder mit schlafwandlerischer Sicherheit durch die komplexen Informationsräume, ohne je ein Wort über die Theorie des Hypertextes gehört zu haben.

Natürlich war das Problem der automatischen Erzeugung von Hypertexten aus herkömmlichen Texten seit langem ein spannendes Thema für die Proponenten des neuen Medientyps (Glushko 1989, Bernstein 1990, Kuhlen 1991, Riner 1991). Das Hauptaugenmerk wurde dabei vorerst aber auf die sinnvolle Partitionierung der Texte in Hypertext-Informationsein- 
heiten und deren automatische Verknüpfung gelegt. Eine solche Transformation erfordert aber ein tieferes Verstehen der inhaltlichen Struktur, als es heute maschinell modellierbar ist.

Aufgehalten wurde diese Entwicklung durch leicht nachvollziehbare ergonomische Beweggründe. Die Zahl der Referenzen pro inhaltstragender Seite sollte überschaubar gehalten werden, damit einerseits die Lesbarkeit nicht beeinträchtigt wird, andererseits aber auch die wesentlichen Referenzen nicht in einem Verknüpfungsdickicht untergehen. In Tutorien und Lehrbüchern zur Herstellung von WWW-Seiten findet man heute noch die Warnung vor einem Übermaß an Hyperreferenzen. Natürlich gelten andere Regeln für Katalog- oder Indexseiten. Hier können auch heute schon alle Begriffe mit Referenzen versehen werden.

Die automatische Verknüpfung mit Zielen außerhalb des Textes war erst einmal auf wenige Situationen beschränkt, in denen Wörterbücher, Glossare oder Terminologien eindeutige Referenzen vorgaben. Eine solche automatische Verknüpfung von Wörtern zu Wörterbucheinträgen bzw. Übersetzungen wurde z. B. im Locolex System der XEROX-Forschung (Bauer et al. 1995) sowie im RichLink System der Firma Sentius verwirklicht. Während Locolex die alternativen Übersetzungen direkt in einem Rollmenü präsentiert, verwendet Sentius eine proprietäre Technologie für die Produktion, Repräsentation und Präsentation der angereicherten Texte. Die Texte können mit einem speziellen Zusatz (plug-in) im Netscape Navigator betrachtet werden. Das Problem der Unübersichtlichkeit der vielen Referenzen und der Vermischung von automatisch angebrachten Nachschlage-Referenzen mit den Hyperreferenzen der Autoren wurde so gelöst, dass die zusätzlich angebrachten Verknüpfungen nicht durch Unterstreichung, sondern nur durch die Cursorform angezeigt werden.

Uszkoreit (1999) argumentiert für eine sehr dichte Vernetzung der Information, in der jede Informationseinheit, das heißt z. B. im Extremfall, dass jedes Wort in einem Text als Anker für mehrere relationale Hyperverknüpfungen dient. Die Frage der Hervorhebung von Text und Referenzen ist hier von der Verknüpfung getrennt. Wie beim RichLink System von Sentius oder wie bei Ankern in Bildern zeigt lediglich die Form des Cursors an, ob Verknüpfungen vorhanden sind. Ziele der Verknüpfungen können erklärende, weiterführende, neue oder alternative Informationen sein. Aber auch Programme oder Geschäftsprozesse können ausgehend von einem Anker parametrisiert aufgerufen werden. Eine solche extreme Verwendung der Hyperverknüpfung ist sicher ganz im Geiste der Erfinder des Hypertextes, wenngleich sie auch so nicht beschrieben wurde. Sie unterstützt die ursprüngliche Idee, weil sie die Umwandlung von Information in wissensähnliche Strukturen konsequent fortsetzt. Das Ziel ist eine digitale Erweiterung des menschlichen Wissens, die zudem kollektiv genutzt und weiterentwickelt werden kann. 


\section{Fusion Viag-Alusuisse perfekt}

HANDELSBLATT, Donnerstag, 26. November 1998

HB DÜSSELDORF. Morgen, spätestens aber für Montag wird die Bekanntgabe des Zusammenschlusses der Münchener Viag AG und der Alusuisse-Lonza AG (Algrawn) Zürich, erwartet. Anfang vergangener Woche war d Firmeninfo Plan für ein neues deutsch-schweizerisches Untern Produkte men mit einem Gesamtumsatz von rund $41 \mathrm{Mrd}$. DI Indikatoren mehr als 100000 Mitarbeitem bekanntgeworden.

Verträge Kontakte Homepage Nachrichten

Abbildung 1. Beispiel einer relationalen Hyperreferenzierung aus Uszkoreit (1999)

\section{Die Hyperverknüpfung im effektiven Informationsmanagement}

In diesem Abschnitt soll demonstriert werden, dass die Hyperverknüpfung ein Grundproblem des modernen Informationsmanagements lösen kann. $\mathrm{Zu}$ diesem Zweck müssen wir zuerst den Begriff der Information und die Aufgabenstellung des Informationsmanagements betrachten.

Uszkoreit (1999) schlägt einen Informationsbegriff vor, der sich nur über die Systeme definiert, die durch Information beeinflusst werden. Information sind Konfigurationen von wiederkehrenden Mustern, die indirekt die Auswahl zwischen möglichen konstitutiven Folgezuständen eines zustandsverändernden Systems beeinflussen. Die Beeinflussung ist indirekt, weil es innerhalb des zustandsverändernden Systems ein Teilsystem geben muss, das durch Evolution, Lernen oder Konstruktion auf die Information vorbereitet sein muss. Die Information kann direkt kausal nur (informationelle) Zustandsübergänge in diesem Teilsystem beeinflussen. Wenn die Information von diesem Teilssystem erkannt wird, beeinflusst dieses wiederum kausal dieAuswahl zwischen möglichen konstitutiven Folgezuständen.

Diese Auffassung steht nicht etwa im Widerspruch zu Shannons (1948) statistischer Informationstheorie, da letztere sich nur auf einen Aspekt der Information bezieht, das wahrscheinlichkeitstheoretisch konstruierte Konzept des Informationsgehalts.

Jede Konfiguration von Energie oder Materie, die aus wiederkehrenden Mustern besteht, könnte so zu Information werden, auch ohne dass sie eine semantische Bedeutung zugewiesen bekommt. Die DNA im Zellkern 
enthält Information, ohne dass wir den genetischen Mustern Denotate zugestehen müssen. Andererseits können Muster, die heute Information sind, diese Eigenschaft verlieren, sobald es keine Systeme mehr gibt, die sich von den Mustern beeinflussen lassen.

Im Informationsmanagement geht es um die Versorgung der Funktionen bzw. Mitarbeiter einer Organisation mit Informationen, die das Arbeitsergebnis der Empfänger sichern und verbessern. Wir wollen hier nur Information betrachten, die geeignet ist, rationale Entscheidungen zu beeinflussen, und den Einfluß von Information auf die emotionalen Zustände oder die Aufmerksamkeit unberücksichtigt lassen.

Das Ziel muss sein, Informationen, die geeignet sind, Entscheidungen zu verbessern, zu sammeln, zu verwalten, aufzubereiten und zu präsentieren. Die Aufbereitung schließt die Sortierung, Strukturierung, Filterung und Zusammanfassung ein. Der Erfolg des Information Management misst sich letztendlich an der Qualität und Quantität der Information sowie am Zeitpunkt und der Art ihrer Präsentation.

Potentielle Information wird also zu echter, zu genutzter Information, wenn sie eine Entscheidung beeinflusst. Wenn der menschliche Entscheider in seinem Arbeitsalltag eine Entscheidung fällen muss, kann es zwei Arten von Information geben, die die Qualität der Entscheidung beeinflussen können.

Zum Einen werden oft Informationen berücksichtigt, von denen von vorherein feststeht, dass sie existieren. Dazu gehören zum Beispiel Alter und Adresse einer Antragstellerin oder Kontostände. In vielen Arbeitssituationen ist die Einbeziehung solcher Informationen in den Entscheidungsprozess vorgeschrieben und wird durch die Aufnahme in Formulare oder Erklärungen erzwungen. Auch wenn die Beschaffung solcher erwarteter Information mitunter aufwendig sein kann, so gibt es doch ein klares Kriterium für den Abbruch der Suche.

Ganz anders stellt sich die Beschaffung von Informationen dar, deren Existenz nicht vorausgesetzt werden kann. Dazu gehören Angaben über mögliche neue Produkte von Wettbewerbern, über bisher unbekannte Insolvenzfälle oder über neueste Erfindungen. In diesen Fällen kann die Informationssuche äußerst aufwendig sein, weil es kein sicheres Abbruchkriterium gibt.

Wenn der Entscheider zu früh informiert wird, müssen die Informationen zwischengespeichert und in der Entscheidungssituation wiedergefunden werden. Die systematische Zwischenspeicherung kann problematisch werden, wenn der Entscheider nicht einmal weiß, in welchem Entscheidungskontext die Nachricht von Bedeutung sein wird.

Muss sich der Entscheider die Information erst zusammensuchen, während der Vorgang in Bearbeitung ist, verzögert sich die Entscheidung. Es kommt auch zu einer erhöhten kognitiven Belastung des Entscheiders, 
weil durch die angestoßene Informationsbeschaffung oft mehrere Vorgänge parallel abgearbeitet werden. Wenn neue Informationen eintreffen, muß der Entscheider sich wieder an die bereits vorliegenden Informationen erinnern, bzw. diese neu abrufen.

In der effizienten Organisation von Produktionsprozessen hat sich eine Methode als sehr erfolgreich erwiesen, die in den japanischen Werken des Automobilherstellers Toyota unter dem Namen Kanban (Harrison 1992) entwickelt wurde. Diese Methode reduziert Logistik- und Lageraufwand, indem der Nachschub an Zulieferguitern wie Material und Teilen immer erst dann erfolgt, wenn die Güter wirklich benötigt werden. In der westlichen Betriebswirtschaft wurde die Methode als JT (für just-in-time) Versorgung bekannt. Die Kanban Methode beruht auf einer lokalen Steuerung durch lokal weitergereichte Bedarfsmeldungen. In diesen werden nicht nur zur Zeit, sondern auch zu Qualität und Quantität genaue Mindestanforderungen formuliert, die dann weder unter- noch überschritten werden. Das Äquivalent im Informationsmanagement wäre die gezielte Informationslieferung auf Anfrage. Nur wenn der Informationskonsument vor einer Entscheidungsaufgabe steht, fordert er die relevante Information an.

Das ist bereits eine Verbesserung gegenüber der Informationslieferung nach Themen durch sogenannte Push-Dienste oder periodische Informationslieferungen. In den noch vor kurzem überschwenglich gepriesenen Push-Diensten werden Informationen zu ausgesuchten Themen an die Benutzer geliefert, sobald sie verfügbar sind. Während ein solcher Dienst sehr sinnvoll ist für Aufgabenbereiche, in denen es darauf ankommt, schnell auf neue unvorhergesehene Entwicklungen zu reagieren, stellt er Bereiche, deren Zeitplanung durch Workflow-Prozesse von außen dirigiert wird, vor das Problem der Zwischenspeicherung und zwingt sie so, ein eigenes Informationsmanagement aufzubauen.

Durch die Möglichkeiten der automatischen Hyperverknüpfung kann das Ideal einer JIT-Informationsversorgung noch besser verwirklicht werden. Bei einer Entscheidung gibt es meistens drei Arten von Information: den Entscheidungsauslöser (decision trigger), die Vordergrundinformation und die Hintergrundinformation. Der Entscheidungsauslöser kann z.B. ein Antrag, eine Anfrage, eine Beschwerde oder ein Beschaffungsvorschlag sein. Die Vordergrundinformation besteht aus den Angaben, die für den Entscheidungsprozess benötigt werden. Hintergrundinformationen sind Angaben, die die Qualität der Entscheidung verbessern können, wenn die Art der Entscheidung und die vorhandene Vordergrundinformation einen Ermessensspielraum offen läßt.

Wenn wir den Informationsauslöser nun zugleich als Bedarfsanmeldung für eine Informationszulieferung betrachten und die relevante Information durch Hyperverknüpfung exakt mit den korrespondierenden Elementen des Entscheidungsauslösers assoziieren, wird die Information so 
bereit gestellt, dass sie in dem Augenblick in den Aufmerksamkeitsbereich des Benutzers gelangt, in dem dieser die Information verwenden kann oder muss.

Hier kann man nun mit einer einfachen Methode erreichen, dass bestimmte Informationen berücksichtigt werden müssen. Durch eine Markierung des Referenzträgers (z.B. Einfärbung) kann darauf verwiesen werden, dass bestimmte Information betrachtet werden muss. Der Abschluss des Vorgangs kann elektronisch blockiert werden, bis die erfolgte Berücksichtigung der Information durch das Klicken auf eine speziell eingefügte Taste bestätigt wurde.

Auch in den traditionellen Arbeitsabläufen hat man bereits versucht, soviel Information wie möglich mit dem Entscheidungsauslöser zu verbinden. Eine typische Art solcher Verknüpfung ist ein Antragsformular. Der Antrag ist der Entscheidungsauslöser, die ausgefüllten Felder enthalten entscheidungsrelevante Vordergrundinformation, einige Felder, wie z. B. ein Feld für weitere Kommentare, mögen auch Hintergrundinformation enthalten. Nur oblag das Ausfüllen der Felder dort der Beabeiterin bzw. dem Antragsteller.

\section{Assoziative Suche aus dem Kontext und lokale Verknüpfungen}

Ein gewichtiger Nachteil heutiger Suchmaschinen ist die unauflösbare Ambiguität vieler Suchausdrücke. Die menschliche Sprache ist hochgradig ambig. Das bezieht sich insbesondere auf ihren Wortschatz. Auch Wörter, die als Namen oder Fachbegriffe verwendet werden, besitzen oft mehr als eine Bedeutung. Der Mensch wird sich bei der Interpretation sprachlicher Äußerungen der Mehrdeutigkeit oft gar nicht bewusst. In dem Kontext des Diskurses wird bei ihm in der Regel nur die intendierte Lesart von Wörtern aktiviert. In der Sprachtechnologie versucht man mit statistischen Verfahren, die kontextgesteuerte Disambiguierung zu modellieren. Für viele Anwendungen lassen sich so schon heute Mehrdeutigkeiten auflösen. Bei der Interpretation von Anfragen an eine Suchmaschine versagen diese Methoden gewöhnlich. Dadurch, dass die mittlere Länge der Suchausdrücke sehr gering ist (etwas über zwei Wörter), ist bei Suchanfragen meist kein ausreichender Kontext vorhanden, um verlässlich disambiguieren zu können. Im Information Retrieval hat man daher verschiedene Möglichkeiten untersucht, um frühere Suchanfragen oder persönliche Interessengebiete bzw. Präferenzen für die Disambiguierung zu nutzen.

Wenn die Suche aber nicht durch die Eingabe von Suchschlüsseln ausgelöst wird, sondern durch Wörter in einem Text, kann der Kontext bei der Filterung bzw. Reihung der gefundenen Dokumente eingesetzt werden. Er kann auch für die sogenannte Anfragenerweiterung verwendet werden, bei der auch verwandte Begriffe in die Suche einbezogen werden. 
Beim Vorgang des automatischen Hyperreferenzierens könnten neben der Einsetzung der bereits vorhandenen Verknüpfungen auch neue Verknüpfungen durch Suchmaschinen gesucht werden. Nun ist das Durchsuchen einer Indexdatenbank des gesamten WWW für jedes Wort in jedem Text wohl nicht durch einen realen Bedarf gerechtfertigt. Wenn der Benutzer in Einzelfällen eine solche Suche anstoßen will, lässt sich das leicht durch einen entsprechenden Menüpunkt im Verknüpfungsmenü vorbereiten. Aber man könnte durchaus in relevanten Repositorien nach Vorkommen des Begriffes suchen, so z. B. in bestimmten Bereichen des Intranet oder gar in Dokumentsammlungen des Benutzers.

Ich möchte diese Möglichkeit an einem Beispiel verdeutlichen. Nehmen wir an, ich fände in einer Wirtschaftsnachricht eine Erwähnung der PBX Technologie. Wenn mir im Moment nicht einfällt, daß PBX im Bereich Telekommunikation für private branch exchange steht, dann kann ich die automatische Hyperreferenz auf die Definition oder eine Einfuihrung in einem Handbuch verfolgen, um eine Erklärung zu erhalten. Wenn ich mich zudem nur noch vage daran erinnern kann, dass ich mit dem Begriff gerade kürzlich konfrontiert wurde, und mir jede Erinnerung an den Zusammenhang fehlt, dann hilft es mir sicher, Verbindungen zu Vorkommen in meinen eigenen Dokumentbeständen zu sehen. So mag der Begriff in einer nur flüchtig gelesenen Email auftauchen, in einem Bericht, den ich aus Zeitmangel noch nicht durchsehen konnte, oder auf der Tagesordnung einer Bereichsbesprechung, die für die kommende Woche in meinem Kalender vorgemerkt ist. Diese Verbindungen können beim automatischen Referenzieren eingefügt werden.

Halten wir also fest, dass die Suche aus dem Kontext heraus in bestimmten Fällen mit der automatischen Hyperreferenzierung sinnvoll verbunden werden könnte. In anderen Fällen wird der Benutzer eine assoziative Suche vielleicht erst beim Lesen des Dokuments anstoßen. Das könnte dann eine Suche sein, die das gesamte zugängliche Web plus Intranet und persönlichen Beständen umfasst oder parallel mehrere Informationsräume durchsucht, während der Benutzer zuschaut und die Suche jederzeit abbrechen oder erweitern kann. Eine Metapher für solch eine interaktive Suche ist das freie Assoziieren über einen Begriff. Über eine Verknüpfung wird die Suche gestartet. Die Ergebnisse werden in einem gesonderten Fenster angezeigt. Das Ergebnis baut sich inkrementell auf. Bei der Anzeige werden die gefundenen Dokumente nach ihrer Herkunft und ihrer Relevanz geordnet. Der Einsatz der Suchmaschinen erlaubt es uns, parallel zu mehreren Begriffen zu suchen oder auch weiterzulesen während die Suche noch andauert. In der Abbildung 2 ist eine solche Suchsituation dargestellt. 


\section{New wireless voice technology introduced \\ Posted at 5:09 PM PT, Feb 8, 1999 \\ By Stephen Lawson, InfoWorld Electric \\ NTT Labs on Monday brought Dick Tracy into the enterprise, introducing a wireless voice and data system that can use a wrist radio at the Demo 99 conference. \\ AirWave technology, demonstrated for the first time in the United States at this week's conference in Indian Wells, Calif., is based on a wireless PBX. Small, handheld phones -- and a wrist radio that looks like an oversized watch -- can be used to make voice calls and exchange data around a building or campus. The handheld phones can be switched to a public cellular mode to become con-}

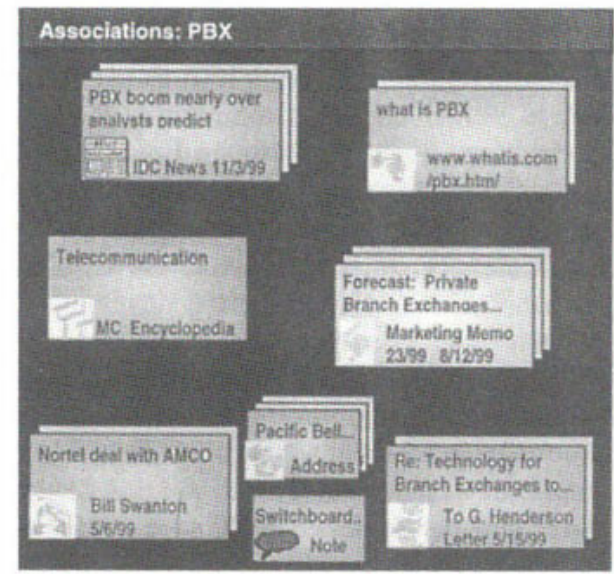

Abbildung 2. Konstruiertes Beispiel einer assoziativen Suche

\section{Hyperverknüpfung und Wissensweitergabe}

Eines der zentralen Ziele des Wissensmanagements ist das Teilen von Wissen (knowledge sharing), d.h. das Überführen von individuellem Wissen in das kollektive Wissen einer Organisation. (Neilson et al. 1997) Im Gegensatz zu Kapital, Personal und Boden ist das Wissen eine Ressource die man nicht verliert, wenn man sie weitergibt, und die nicht abnimmt, wenn man sie teilt. Die Ressource Wissen hat hingegen sogar die besondere Ei- 
genschaft, sich schneller zu vermehren, je öfter man sie verwendet. Wenn Personen oder Abteilungen einer Firma jedoch Erfahrungen machen, die für den wirtschaftlichen Erfolg der gesamten Firma wichtig sind, dann wird dieses Wissen oft nicht weitergegeben.

Dafür gibt es mehrere Gründe:

1. Das durch Erfahrung erworbene Wissen bedeutet oft einen persönlichen Vorteil im innerbetrieblichen Wettbewerb, den man ungerne aufgibt.

2. Die Aufzeichnung der Erfahrung ist eine Extraleistung, die nicht vergütet wird.

3. Besonders bei überregional oder global operierenden Organisationen gibt es oft keine Mechanismen der Weitergabe der aufgezeichneten Erfahrungen an die Kolleginnen oder Unterorganisationen, die von dem Wissen profitieren könnten.

Im Wissensmanagement hat man verschiedene Mechanismen getestet, um die Weitergabe von Wissen zu belohnen. Da man nicht einen jeden einzelnen Hinweis an die Kollegen mit einer Prämie vergüten kann, führt z. B. die wiederholte Weitergabe von Erfahrungen über das Intranet oder innerbetriebliche Email-Gruppen zur Erlangung eines Experten-Status. Dieser wiederum wirkt sich bei Prämien, Gehaltseinstufungen oder Beförderungen aus. Hier stellt sich jedoch ein nicht zu unterschätzendes Problem: Wenn die häufige Weitergabe von Information zu einem Thema belohnt wird, wie vermeidet man dann die Verbreitung von nutzloser oder redundanter Information. Es ist nicht so sehr die Ungerechtigkeit einer ungerechtfertigten Belohnung, die man vermeiden muss, wie vielmehr die Belastung der anderen Mitarbeiter mit unnützer oder gar irreführender Information. Das Problem ließe sich durch eine Bewertung der geteilten Erfahrungen nach ihrer Nützlichkeit lösen. Eine solche Bewertung müsste von den Adressaten des weitergegebenen Wissens durchgeführt werden. Sie könnte auch verwendet werden, um veraltete Hinweise zu löschen, bzw. zwischen alternativen Ratschlägen zu entscheiden. Aber auch bei der Einführung von Mechanismen zur Evaluation stellt sich wieder das Problem der erwarteten Mehrleistung und damit das der Motivation der Mitarbeiter.

Die vorgeschlagene Lösung stützt sich wieder auf die Hyperverknüpfung. Wenn eine Mitarbeiterin ihren Kollegen, gewonnene Einsichten, zum Beispiel eine Warnung, zur Verfügung stellen will, dann verfasst sie den Text als einen Kommentar zu ihren eigenen Aufzeichnungen bzw. ihrem Vorgang. Dieser Kommentar wird mit den relevanten Elementen in der Vorgangsbeschreibung verknüpft. Die Verknüpfung ermöglicht es, die Aufzeichnung kürzer zu halten, weil sie eine ausführliche Beschreibung der Situation erspart. Die Verknüpfung wird dann dem Informationsmanagement zur Verfügung gestellt. Bei der automatischen Hyperreferenzierung ähnlicher Vorgangsbeschreibungen, bzw. Entscheidungsauslöser, die 
von anderen Personen bearbeitet werden, wird auch die Warnung an der relevanten Stelle als eine Hyperreferenz eingefügt. Wenn die Warnung oder ein Hinweis besonders wichtig sind, können diese Arten von Hyperreferenzen durch optische Markierungen (z. B. Farbe der Unterstreichung) hervorgehoben werden. Im Gegensatz zur Verbreitung von Hinweisen und Warnungen über Email wird das geteilte Wissen hier nur in einer potenziellen Bedarfssituation angeboten. Im Gegensatz zur internen Veröffentlichung über das Intranet erfordert die Nutzung keinen Suchaufwand. Wenn die Menge an geteilter Erfahrung zunimmt, können Hinweise im Intranet auch leicht übersehen oder vegessen werden.

Damit ist aber erst das Problem der gezielten Weitergabe erledigt. Die andere große Herausforderung ist die Entwicklung eines Mechanismus zur Bewertung der geteilten Erfahrungen. Die extrem einfache Lösung, die ich vorschlagen möchte, ist sofort und ohne großen Aufwand umsetzbar. Ich gehe von drei Prämissen aus:

1. Eine komplexe wortreiche Beurteilung ist unnötig und kontraproduktiv, weil sie wiederum von Menschen ausgewertet und in Prädikate auf einer einfachen Skala übersetzt werden müsste. Deshalb genügt eine binäre oder ternäre Bewertung (maximal eine Werteskala von 1-5).

2. Die Bewertung darf keinen zusätzlichen Arbeitsschritt kosten.

3. Nach dem Öffnen und Lesen des Kommentars ist genau ein Arbeitsschritt, nämlich ein einfacher Klick erforderlich, um zurück in den eigentlichen Arbeitsablauf zu gelangen.

Damit liegt die Lösung auf der Hand: Wenn ein Fenster mit einem zu bewertenden Dokument geöffnet wird, dann werden die normalen Mechanismen zur Rückkehr (Wahlfeld, Menüpunkt und Tastenkürzel) deaktiviert. Am Ende der zu bewertenden Seite finden sich zwei bis fünf Wahlfelder, die jeweils einem Bewertungsprädikat entsprechen. Durch einen Mausklick auf dem ausgewählten Feld wird die Bewertung registriert und die Rückkehr in das Hauptfenster bewirkt. Die Bewertung wird in einem gesonderten Argument der Hyperverknüpfung gespeichert.

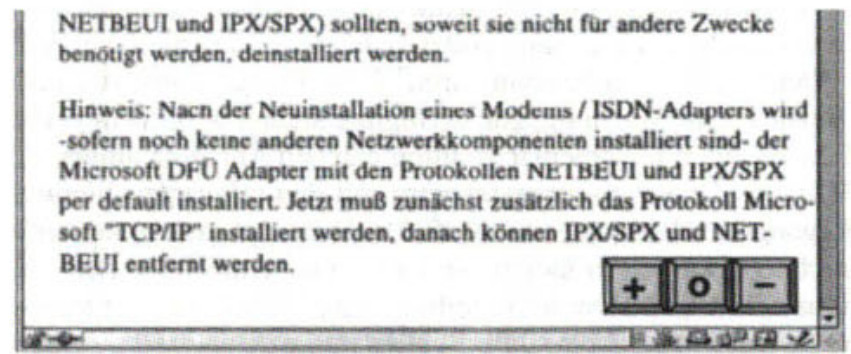

Abbildung 3. Bewertungstasten 
Im Vergleich mit anderen Möglichkeiten der Nutzung und Bewertung (mit)geteilten Wissens bietet die direkte Assoziation der Hinweise mit konkreten Vorgängen eine weitaus bessere Grundlage für die empirische Auswertung der Wissensnutzung und dient damit der Bereicherung des Wissens um neue Erfahrungen. Da wir für jeden neuen Vorgang sowohl die Bewertung des Hinweises als auch die wesentlichen Parameter des Vorgangs kennen und gemeinsam festhalten, eignet sich die resultierende Dokumentation der Vorgänge für die detaillierte statistiche Auswertung.

\section{Sprachtechnologien für die automatische Hyperverknüpfung}

Durch bittere Erfahrung haben die Computerlinguisten einsehen müssen, dass das maschinelle Verstehen beliebiger Texte mit den heutigen technologischen Mitteln nicht realisiert werden kann und auch noch lange ein Traum bleiben wird. Selbst wenn die Computerlinguistik heute bereits in der Lage wäre, alle bekannten Regeln und verborgenen Regularitäten der Sprache in einer formalen Grammatik zu beschreiben und auf dem Computer zu implementieren, so fehlte doch noch die Modellierung des Wissens und der Inferenzen, die der menschliche Sprachbenutzer benötigt, um Texte wirklich zu verstehen.

Dürfte man den Gegenstandsbereich der zu verarbeitenden Texte so stark einschränken, dass eine vollständige Modellierung des Wissens in diesem eingeschränkten Gegenstandsbereich möglich wäre, dann bestünde eine gute Chance, die Texte grammatisch zu analysieren und semantisch interpretieren zu können. Da man aber unter normalen Umständen die Kreativität von Textautoren nicht auf das winzige Terrain einer modellierbaren Miniwelt beschränken kann, gibt es derzeit kaum realistische Anwendungen für das Textverstehen.

Um dennoch automatisch Information aus Texten gewinnen zu können, wurde aus einem Bündel von Methoden eine Technologie entwickelt, die man als Informationsextraktion (information extraction) bezeichnet. (Jacobs 1997) In der Informationsextraktion setzt man sogenannte flache Verfahren der sprachlichen Analyse ein, um zielgerichtet Ausdrücke zu finden, die ganz bestimmte Sachverhalte widergeben. Diese Ausdrücke werden dann in die Felder vorgefertigter Schablonen (templates) eingesetzt. Diese Schablonen entsprechen mehr oder weniger komplexen Datenbankkeinträgen. So kann es bei der Auswertung von Wirtschaftsnachrichten eine Schablone für Personalwechsel im Management geben. Eine solche Schablone braucht mindestens je ein Feld für die Firma, die Position, den bisherigen Inhaber der Stellung und den neuen Stelleninhaber. Mit einer entsprechend komplexen Grammatik könnte man auch versuchen, den Grund für den Wechsel oder die bisherige bzw. zukünftige Fir- 
ma der beiden Führungskräfte zu entdecken. Neben der Auswertung von Personalnachrichten wurde die Informationsextraktion unter anderem für die Sammlung von Informationen über terroristische Anschläge, Firmenzusammenschlüsse oder Mängelberichte verwendet.

Ein Beispiel für eine Anwendung der Informationsextraktion ist das System zur Erkennung von Gewinn- und Umsatzzahlen, das an Forschungsbereich Sprachtechnologie des DFKI auf der Basis des generischen Extraktionssystems SMES entwickelt wurde (Neumann et al. 1997). Hier müssen die Firmenbezeichnungen erkannt werden. Dann müssen die Zahlen für Umsätze und Gewinne korrekt den Zeitintervallen zugeordnet werden.

Die einfachste aber auch wichtigste Analyseleistung betrifft die Erkennung von Namen von Personen, Firmen, Orten oder Produkten. Etwas anspruchsvoller ist die Zuordnung von Preisen zu Produkten, von Produkten zu Firmen, von Personen zu Funktionen oder von Ereignissen zu Orten. Je mehr Felder in einer komplexen Schablone zu füllen sind, desto unzuverlässiger wird das System. In manchen Anwendungen spielt es keine Rolle, ob man wirklich alle Vorkommen der gesuchten Relation findet. Das kann der Fall sein, wenn die Informationsquellen sehr redundant sind, weil mehrere Zeitungen oder Nachrichtenagenturen ausgewertet werden. In einer solchen Anwendung kann man in Zweifelsfällen Felder ungefüllt lassen.

In anderen Anwendungen möchte man alle Vorkommen der Relation finden, stört sich aber nicht so sehr an fehlerhaft gefüllten Feldern. Zu diesen Anwendungen gehören Systeme, deren Ausgabe von Menschen nachbearbeitet wird, denen man zwar die Überprüfung der gefüllten Schablonen, nicht aber die erneute Sichtung aller ausgewerteten Texte zumuten kann.

Ganz zu unrecht wird die Informationsextraktion im Alltag der Computerlinguistik oft als eine wissenschaftlich unergiebige Übergangstechnologie angesehen. Wenn in den meisten IE-Systemen nur sehr einfache Grammatiken und noch einfachere semantische Tests zum Einsatz gelangen, dann liegt das sicher nicht am Wesen der Anwendung, sondern am Fehlen effizienter Grammatiken, die auch partielle Analysen generieren können. Die Informationsextraktion ist eine ideale Anwendung für den Brückenschlag zwischen theoretischer und angewandter Forschung, weil hier ein permanenter Bedarf an schrittweisen Verbesserungen der Erkennungsleistung besteht und weil sich an der Performanz abmessen läßt, inwieweit sich die tieferen Methoden bereits für den praktischen Einsatz eignen.

Die Zukunft wird der Kombination von flachen und tiefen Methoden gehören. So kann man die Wortartenerkenner, Lemmatisierer und flachen Phrasengrammatiken, die meist als Mengen oder Kaskaden regulärer Ausdrücke bzw. der äquivalenten endlichen Automaten formuliert sind, zur Erkennung der relevanten Kontexte verwenden. Die tiefen Grammatiken 
kann man dann zuschalten, wenn es in einem relvanten Kontext um die korrekte Füllung der Felder in den Schablonen geht.

Sind die Verfahren einer verbesserten Informationsextraktion dann nicht doch Kanonen, die man bei der Aufgabe der automatischen Hyperverknüpfung auf harmlose Spatzen richtet? Wohl kaum. Wenngleich es vorerst einmal um die Erkennung von Wörtern und festen Mehrwortausdrücken geht so benötigt man die Maschinerie schon jetzt für die Disambiguierung. Ein Beispiel möge verdeutlichen, dass die Disambiguierung schwer ist und ohne semantische Heuristiken auch nicht immer gelingen kann.

- Stadt: die Stadt Essen -- er ging nach Essen -- er kam von Essen

- Person: G. v. Essen -- Gustaf von Essen

- Firma: CTB Bank von Essen GmbH

- Nomen: das Essen fällt ihr noch schwer -- er redet ständig von Essen

In der Zukunft wird man sicher komplexere Verknüpfungen aufbauen wollen. In den beiden folgenden Beispielen wäre eine Verknüpfung der Wörter „Umsatz" und „Vorjahresergebnis“ mit den jeweiligen Umsatzzahlen von Genentech hilfreicher als simple Verweise auf Wörterbucheinträge:

- Die Firma Genentech lag im Umsatz unter den Vorjahresergebnis.

- Die Firma Genentech lag im Gegensatz zu den anderen Biotechnologieunternehmen im Umsatz unter dem Vorjahresergebnis

In der automatischen Hyperverknüpfung sind dem technologischen Ehrgeiz keine Grenzen gesetzt. Je besser die Analsyseleistungen der Sprachtechnologie werden, desto informativer werden auch die Hyperverknüpfungen.

\section{Heutige Situation und Ausblick}

Es gibt heute in der Computerlinguistik nur sehr wenige Projekte, die sich mit der automatischen Hyperverknüpfung beschäftigen. Einige erfolgversprechende Projekte wurden eingestellt, so zum Beispiel ein Gemeinschaftsvorhaben der Apple-Forschung mit der Brandeis University (Pustejovsky et al. 1997). in diesem Projekt ging es zwar nicht um die dichte Hypervernetzung, aber um den Einsatz von Methoden der lexikalischen Semantik für eine konkrete praktische Anwendung, die Dokumentation des Onlinehilfe Apple Guide.

Im Forschungsbereich Sprachtechnologie am DFKI wurden von Gregor Erbach, Diana Raileanu und Andrew Bredenkamp zwei Parser entwickelt, die Ankerausdrücke erkennen und die relationalen Hyperverknüpfungen einfügen. Die Verknüpfungen werden aus einer von Hand gepflegten Verknüpfungsdatenbank entnommen. Die Methode der Inte- 
gration von relationalen Hyperverknüpfungen und Verknüpfungsmenüs in HTML Dokumente wurde von dem Autor gemeinsam mit Jakob Uszkoreit entworfen und von letzterem in Javascript implementiert. Die um Hyperverknüpfungen angereicherten Seiten können ohne spezielle Zusätze in einem üblichen Browser dargestelit werden. Gegenwärtig arbeiten wir an einem Auftrag einer großen deutschen Bank, dessen Ziel eine Hyperreferenzierung für eine umfangreiche Softwaredokumentation ist.

In den vergangenen Monaten zeigt sich in der Internetindustrie ein verstärktes Interesse an Technologien für die automatische Verknüpfung. Eine gemeinsame Entwicklung von Lotus und Sentius deutet auf eine größere Verbreitung der RichLink Technologie hin. Zudem bietet die neue Firma Flyswat einen gleichnamigen Dienst für die automatische Anreicherung von Web-Dokumenten mit Hyperverknüpfungen an, der ebenfalls auf einer proprietären Technologie beruht.

Mit der zunehmenden Verfügbarkeit von Metakatalogen, Online-Enzyklopädien and anderen Orientierungs- und Nachschlagediensten wird der Bedarf an diesen Technologien noch zunehmen. Auch die Softwarehersteller, die sich auf Produkte für das Wissensmanagement und die Organisation von Arbeitsabläufen spezialisieren, werden nach Möglichkeiten suchen, die Informationsvernetzung für konkrete Anwendungen zu automatisieren. Eine besondere Bedeutung wird in diesem Zusammenhang neuen Methoden und Werkzeugen für die Herstellung von Firmenontologien zukommen. In solchen Ontologien werden die firmen- und aufgabenspezifischen Verknüpfungstypen definiert werden.

Die Einführung der XML Standards, besonders der Standards XLink und XPointer für die Hyperverknüpfung, wird die Zuordnung, Repräsentation, Verwaltung und Darstellung der Verknüpfungen erheblich erleichtern. Die Verknüpfungen können dann wirklich unabhängig von den Texten gehalten und verwaltet werden. So kann z. B. der gleiche Text auf dem Intranet je nach Leser und Arbeitszusammenhang ganz andere Verknüpfungen enthalten. Die XLink Verknüpfungen können zudem echt bidirektional sein und sie können mehr als zwei Informationseinheiten miteinander verbinden.

Es ist abzusehen, dass der zweiten Welle der Hypertextrevolution, die zur dichten Informationsvernetzung führt, noch eine dritte Welle folgen wird, deren Ergebnis ein Reichtum an dynamischen, sich überlappenden Ontologien sein wird, die dem engmaschigen Informationsnetz unzählige parallel existierende Strukturierungen überlegen.

Erst dann wird sich die Vision von Bush, Nelson, Engelbart und den anderen Wegbereitern der Revolution erfüllen: die Hypermedien werden nicht nur im metaphorischen Sinne als Erweiterungen des menschlichen Wissens akzeptiert werden. Sie werden beim kreativen Gebrauch des Wissens eine wichtige Funktion einnehmen und in dieser Funktion auch täglich verwendet werden. Die Sprachtechnologie - und damit auch die 
Sprachwissenschaft - wird bei dieser Entwicklung eine zentrale Rolle spielen.

\section{Literatur}

Bauer, D., F. Segond and A. Zaenen (1995): LOCOLEX: Translation Rolls off Your Tongue. In: Proceedings of the Conference of the Association for Computers and the Humanities and the Association for Literary and Linguistic Computing (ACH-ALLC'95), Santa Barbara, USA.

Bernstein, M. (1990): An Apprentice That Discovers Hypertext Links. In: Proceedings of ECHT ' 90 .

Bush, V. (1945): As we may think. Atlantic Monthly 176, S. 101-108.

Engelbart, D. C. (1963): A conceptual framework for the augmentation of man's intellect. In P. W. Howerton \& D. C. Weeks (Eds.), Vistas in information handling, Vol. 1 (pp. 1-29).

Washington, DC: Spartan Books.

Glushko, R. J. (1989): Transforming Text Into Hypertext For a Compact Disc Encyclopedia, In: Proceedings of CHI '89, ACM Press.

Harrison, A. (1992): Just-in-time manufacturing in perspective, New York, Prentice Hall.

Jacobs, P. (1997) Text Interpretation: Extracting Information. In: R. A. Cole, J. Mariani, H. Uszkoreit, A.Zaenen, V. Zue (eds.): Survey of the State of the Art in Human Language Technology, Cambridge University Press and Giardini.

Kuhlen, R. (1991): Hypertext. Ein nicht-lineares Medium zwischen Buch und Wissensbank. Berlin, Heidelberg, New York.

Neilson, R.E. (1997): Collaborative technologies and organizational learning, Hershey Idea Group Publishing.

Nelson, T. (1965): The Hypertext. In: Proceedings of the World Documentation Federation.

Neumann G., R. Backofen, J. Baur, M. Becker and C. Braun (1997): An Information Extraction Core System for Real World German Text Processing. In: Proceedings of 5th ANLP, Washington.

Pustejovsky, J., B. Boguraev, M. Verhagen, P. Buitelaar,. and M. Johnston (1997): Semantic Indexing and Typed Hyperlinking. In: Proceedings of the American Association for Artical Intelligence Conference, Spring Symposium, NLP for WWW ... Stanford University, CA, 120-128.

Riner, R. (1991): Automated Conversion. In: Hypertext/Hypermedia Handbook (eds. Berk, E. and Devlin, J.), Intertext Publications/McGraw Hill Publishing Co., Inc., New York.

Shannon (1948) : A Mathematical Theory of Communication. In: Bell Syst. Tech. J. Uszkoreit, H. (1999): Sprachtechnologie für die Wissensgesellschaft: Herausforderungen und Chancen für die Computerlinguistik und die theoretische Sprachwissenschaft. In: Meyer-Krahmer, F. und S. Lange (eds.): Geisteswissenschaften und Innovationen, Physica Verlag. 